\title{
Obtención de un Índice General de Seguridad en Pistas Aeroportuarias mediante un Proceso de Ponderación de Criterios Basado en Datos Cardinales
}

\author{
Tiago J. M. Gonçalves y Anderson R. Correia \\ Departamento de Engenharia Aeronáutica e Mecânica, Instituto Tecnológico de Aeronáutica (ITA), \\ Praça Marechal Eduardo Gomes, 50, 12228-900, São José dos Campos, São Paulo - Brasil. \\ (e-mail: tiagojmg@ita.br; correia@ita.br)
}

Recibido Oct. 28, 2014; Aceptado Dic. 26, 2014; Versión final Feb. 19, 2015, Publicado Ago. 2015

\begin{abstract}
Resumen
Este trabajo propone un enfoque para obtener un índice global de seguridad operacional en pistas aeroportuarias, con criterios basados en eventos de seguridad. Se ponderan los eventos en base a los datos cardinales extraídos a partir de la percepción de un experto sobre los riesgos de seguridad derivados de ellos, utilizando el método de los pesos dinámicos (Swing Weights). Se ha utilizado la teoría del valor multiatributo para agrupar los diferentes criterios en un índice global, con el fin de analizar la evolución de los niveles de seguridad. Este enfoque se aplicó a las operaciones del Aeropuerto Internacional de São PauloGuarulhos. Se realizaron análisis de tendencias con el fin de poner de relieve los indicadores con mayor urgencia para llevar a cabo acciones encaminadas a aumentar los niveles de seguridad. Los resultados fueron considerados consistentes, lo que contribuye a la continuidad de su aplicación práctica en la gestión de la seguridad del aeropuerto.
\end{abstract}

Palabras clave: seguridad; aeropuertos; teoría del valor multiatributo; decisión; indicadores de seguridad

\section{Obtaining a Global Index of Operational Safety in Airport Runways through a Process of Weighting of Criteria based on Cardinal Data}

\begin{abstract}
This paper proposes an approach to obtain a global index of safety in airport runways, with criteria based on safety events. Events were weighted based on the cardinal data extracted from the perception of an expert on safety risks, using the Swing Weights method. The multi-attribute value theory to group the different criteria in a global index was employed to analyze the evolution of the safety levels. This approach was applied to the operations of the São Paulo-Guarulhos International Airport. Trend analyses were done to highlight the most urgent indicators to carry out actions to increase the safety levels. The results were considered to be consistent, contributing to the continuity of its practical application in the management of airport safety.
\end{abstract}

Keywords: safety; airports; multi-attribute value theory; decision; safety indicators 


\section{INTRODUCCIÓN}

Un estudio realizado por Boeing (2011) llegó a la conclusión de que aproximadamente el $15 \%$ de los accidentes mortales de aviación comercial se producen en el sistema de pistas aeroportuarias, lo que pone de relieve la importancia de contar con metodologías adecuadas para evaluar y mejorar los resultados de la seguridad operacional en este sistema. De acuerdo con Roelen y Klompstra (2012), la eficacia de un sistema de seguridad aeroportuaria se controla por medio de indicadores de eficacia de la seguridad. Estos indicadores miden los cambios en el nivel de seguridad a lo largo del tiempo con el fin de orientar las acciones dirigidas a la reducción de riesgos (Janaćković et al., 2011). Øien et al. (2011) afirman que los principales objetivos de los indicadores de eficacia son: (1) controlar el nivel de seguridad de un sistema; (2) respaldar la toma de decisiones en materia de cómo y dónde actuar para mejorar la seguridad operacional; y (3) motivar a los actores con poder de decisión para que tomen las medidas necesarias en vías de mantener y mejorar la seguridad operacional.

Øien et al. (2011) sostienen que los indicadores de seguridad operacional pueden clasificarse como indicadores leading o lagging. Los indicadores lagging utilizan el número de accidentes, incidentes o cuasi accidentes como datos de análisis. Por su parte, los indicadores leading siguen de cerca los acontecimientos de menor gravedad que puedan producirse en el sistema antes de que falle. A pesar del gran debate existente sobre el mejor tipo de indicador, aún no se ha llegado a un consenso sobre este tema (ver Allford, 2009; Hale, 2009; Hopkins, 2009a, 2009b, Wreathall, 2009). Además, la OACl (2009) establece que se deben utilizar métodos reactivos, proactivos y predictivos de manera coordinada para lograr los objetivos de seguridad aeroportuaria. Esta posición fue ratificada por Hopkins (2009a), quien argumentó que la distinción entre ambos tipos de indicadores no es clara y, por tanto, carece de importancia.

Tradicionalmente, se utilizaban las tasas de accidentes como indicadores para evaluar la eficacia en materia de seguridad pero, una vez que aumentó la seguridad y los accidentes se hicieron poco frecuentes, se hizo patente la necesidad de contar con una mayor base de datos estadísticos, lo que dificultó el procedimiento de evaluación (Roelen y Klompstra, 2012). Como resultado, comenzaron a utilizarse los datos sobre incidentes en los análisis de seguridad.

Según Reason (1997), la ventaja de recopilar y analizar datos sobre incidentes es que ofrecen lecciones, como: (1) si se obtienen las conclusiones correctas y se ponen en práctica, éstas actúan como "vacunas" para movilizar las defensas del sistema de seguridad contra sucesos más graves en el futuro y, al igual que las vacunas, lo hacen sin producir daños a nada ni nadie del sistema; (2) los incidentes facilitan información cualitativa sobre la forma en que una serie de pequeños fallos de seguridad pueden unirse y alinearse, y terminar ocasionando grandes desastres; (3) se producen con mayor frecuencia que los accidentes, desastres y otros acontecimientos de mayores proporciones, facilitando de tal manera las cifras necesarias para llevar a cabo análisis cuantitativos más avanzados; y (4) los incidentes recuerdan con crudeza los peligros a los que se enfrenta el sistema.

Para poder realizar un control de la seguridad de un sistema aeroportuario es necesario desarrollar índices numéricos que permitan comparar el nivel de eficacia de diferentes períodos, lo cual permite, a su vez, comprobar la eficacia del sistema a lo largo del tiempo, realizar acciones de evaluación comparativa y establecer objetivos de mejora. Estos índices están formados por la suma de diferentes indicadores de seguridad operacional, que forman un índice global de seguridad. Además, tal y como indica la ANAC (2009), los indicadores de seguridad operacional deben sopesarse en proporción a los riesgos que suponen para la seguridad. Lobianco y Correia (2013) propusieron una metodología empírica basada en la realización de una media ponderada del número de acontecimientos de cada tipo de Evento de Seguridad Operacional (ESO) producidos en un período determinado, arbitrando los pesos basándose en la matriz de calificación de riesgos definidos por la OACI (2009).

La ANAC (2009) define dichos ESO como cualquier accidente, incidente, acontecimiento o condición peligrosa con el potencial de causar daños, lesiones o poner en peligro la viabilidad de la operación de un aeropuerto. La falta de base teórica de este estudio reveló la necesidad de adoptar un nuevo enfoque, utilizando modelos capaces de proporcionar resultados más representativos de la realidad y cuyos cálculos estén defendidos por fundamentos que puedan esgrimirse ante los responsables de la toma de decisiones.

En la búsqueda de una base teórica, Gonçalves Correia y Lobianco (2014) propusieron el uso de la Teoría del Valor Multiatributo (del inglés Multi-Attribute Value Theory - MAVT) (Goodwin y Wright, 2009) para obtener el índice general de seguridad, basándose en las teorías de apoyo a las decisiones multicriterio. La MAVT no utiliza pesos, sino tasas de sustitución, que se calcularon utilizando el método de Rank Order Centroid - ROC (Ahn, 2011; Barron y Barrett, 1996), basado en las tres clases de riesgos (inaceptables, aceptables siempre que se mitiguen y aceptables) definidos en la matriz de clasificación adoptado por la 
OACI (2009). El uso de estas tres clases dio como resultado tres valores para representar las tasas de sustitución de todos los criterios, lo que permitió realizar una pequeña diferenciación entre las tasas, concluyéndose que el cálculo de las tasas de sustitución con este procedimiento sólo devuelve tres valores posibles para representar las tasas de todos los criterios, lo cual fue considerado insuficiente para describir exhaustivamente toda la diferencia entre los criterios analizados.

Los trabajos relacionados con la obtención de un índice global de seguridad en pistas aeroportuarias, anteriormente presentados en la literatura, únicamente utilizan datos ordinales de la matriz de clasificación de riesgos de la OACI (2009) para llevar a cabo la ponderación de los criterios. Esta característica ordinal de los datos en la que se basa la ponderación de los criterios reduce la exactitud de los resultados, siendo deseable que dichos datos faciliten no sólo el orden de importancia de los criterios, sino también el grado en que cada criterio es más (o menos) importante que los demás. Por lo tanto, existe un vacío en la literatura en lo referente a la forma de abordar este problema en la presentación de datos cardinales sobre las tasas de sustitución de cada criterio. Estos datos cardinales se puede obtener a partir de juicios de un experto en seguridad aeroportuaria, lo que haría posible determinar con mayor precisión las tasas de sustitución utilizadas y, a su vez, resultar en la consecución de valores más fiables para el índice global de seguridad en pistas aeroportuarias.

En este contexto, el presente trabajo tuvo como objetivo desarrollar un modelo para obtener un índice global de seguridad operacional en pistas aeroportuarias basado en ESO y cuyos criterios sean ponderados mediante el uso de datos cardinales relativos a los riesgos aeroportuarios, extraídos del juicio de expertos del sector. Además, este trabajo tiene los siguientes objetivos concretos: (i) obtener escenarios para la presencia de los ESO analizados, con el fin de respaldar los juicios para la ponderación de los criterios; (ii) realizar un análisis de la eficacia local y global de la seguridad aeroportuaria con el fin de identificar tendencias y diagnosticar problemas; y (iii) presentar recomendaciones para respaldar la planificación de acciones encaminadas a elevar los niveles de seguridad aeroportuaria.

Por último, hay que destacar que para hacer posible mejorar un sistema logístico, es necesario identificar los aspectos del sistema se deben mejorar. Por lo tanto, es necesario llevar a cabo una evaluación del desempeño de los aspectos del sistema, con el fin de identificar aquellos con la más alta prioridad para la mejora. La literatura presenta varios trabajos sobre la evaluación del desempeño, como Campos et al. (2004) y Chalmeta y Matilla (2007).

\section{METODOLOGÍA}

La Fig. 1 muestra el modelo propuesto en este trabajo para calcular un índice global y evaluar la seguridad de las pistas aeroportuarias basado en ESO. En este modelo, se utilizan los diferentes ESO registrados en la base de datos (BD) para definir los criterios y se usa la MAVT (Goodwin y Wright, 2009) para obtener un índice global de la seguridad en las pista aeroportuarias.

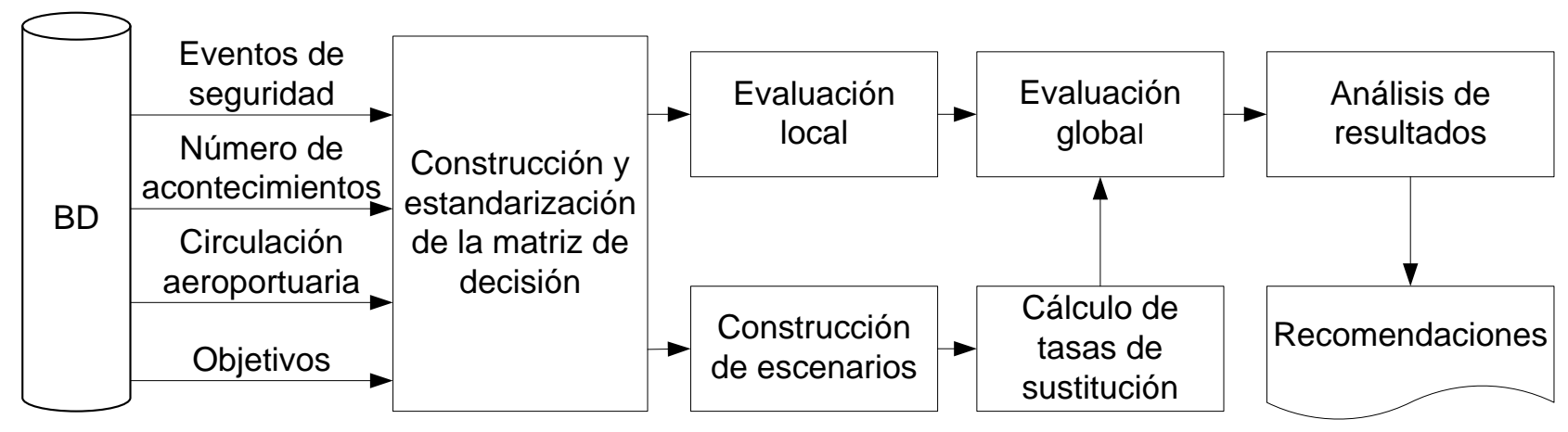

Fig. 1: Modelo para la evaluación de la seguridad operacional aeroportuaria.

En la primera etapa, se elabora una matriz de decisión $D=\left(q_{i j}\right)_{m \times n}$ a partir del historial de ESO. En ella, se utiliza cada tipo de ESO como un criterio y los períodos analizados como alternativas evaluadas. Debido a los cambios ocurridos en el movimiento aeroportuario a lo largo del tiempo, Lobianco y Correia (2013) afirman que se debe incluir en la matriz de decisión el número de ESO sucedidos por cada 100.000 aeronaves en circulación, calculado de acuerdo con la ecuación 1. 


$$
q_{i j}=\frac{100.000 \times Q_{i j}}{m_{i}}
$$

En la Ecuación 1, $q_{i j}$ representa el número de acontecimientos del evento $j$ en el año $i$ por cada 100.000 aeronaves en circulación; $Q_{i j}$ el número de acontecimientos del evento $j$ en el año $i$; y $m_{i}$ es el número de aeronaves en circulación en el año $i$.

Según Basso et al. (2004), un sistema de seguridad aeroportuaria debe fijar objetivos para la prevención de accidentes. Cuando se alcanzan los objetivos establecidos en la política de seguridad operacional, deben definirse objetivos más estrictos con el fin de elevar los requisitos de seguridad a un nuevo nivel. En este sentido, para modelar los objetivos de seguridad aeroportuaria, la MAVT ofrece dos puntos de referencia, denominados niveles "neutro" y "bueno", que se utilizan para estandarizar los valores de la matriz de decisión. Una eficacia por debajo del nivel "neutro" se considera comprometedora, mientras que una eficacia entre los niveles "neutro" y "bueno" se considera competitiva y una eficacia por encima del nivel "bueno" se considera excelente.

Según Edwards y Barron (1994), la estandarización de la matriz de decisión se puede realizar utilizando la Ecuación 2. En ella, xij es el número de ESO sucedidos por cada 100.000 aeronaves en circulación con respecto al i-ésimo período y al j-ésimo criterio, $\mathrm{N}_{\mathrm{j}}$ es el valor de la eficacia en el nivel "neutro" del j-ésimo criterio, $\mathrm{B}_{\mathrm{j}}$ es el valor de la eficacia en el nivel "bueno" del j-ésimo criterio e $\mathrm{y}_{\mathrm{ij}}$ es el valor de la eficacia local resultante en relación al i-ésimo período y al j-ésimo criterio.

$$
y_{i j}=\frac{100\left(N_{j}-x_{i j}\right)}{N_{j}-B_{j}}
$$

Una vez realizada la estandarización de la matriz de decisión, se procede a la evaluación local. Esta evaluación consiste en analizar la existencia o no de tendencias de disminución de los valores de eficacia para cada criterio, lo que permite planificar acciones para elevar el nivel de seguridad desempeñado por él mismo.

Junto a la evaluación local, se lleva a cabo la determinación de las tasas de sustitución de los criterios. Según ANAC (2009), los indicadores de seguridad deben ponderarse en proporción a los riesgos que suponen para la seguridad. Por lo tanto, la FAA (2006) señala que el riesgo de un ESO debe usar como referencia su presencia en el peor escenario posible del sistema analizado. En este sentido, para fundamentar la ponderación de los criterios, se propuso la construcción de escenarios pesimistas correspondientes a los ESO en los que se basa la definición de cada criterio, basándose en la percepción de un experto. Una vez descritos estos escenarios, cada uno de ellos correspondiente a la ocurrencia de un ESO asociado a un criterio, se utiliza el método Swing Weights (MONTIBELLER et al., 2006) para obtener las percepciones de un experto y ponderar los criterios.

En el siguiente paso, se utiliza una Función de Valor Multiatributo (Multi-Attribute Value Function - MAVF), que forma parte de la MAVT, para llevar a cabo una evaluación global y calcular el índice global de seguridad para pistas aeroportuarias. Según Belton (1999), la MAVF más ampliamente utilizada es la Función de Agregación Aditiva, mostrada en la Ecuación 3, que se utilizó en este trabajo.

$\mathrm{V}(\mathrm{a})=\sum_{\mathrm{i}=1}^{\mathrm{n}} \mathrm{w}_{\mathrm{i}} \times \mathrm{v}\left(\mathrm{c}_{\mathrm{i}}\right)$

En la Ecuación 3, wi representa la tasa de sustitución del criterio i, v( $\left.\mathrm{c}_{\mathrm{i}}\right)$ es el valor de eficacia de la acción a en el criterio i (obtenido de la matriz de decisión estandarizada), $n$ es el número de criterios del modelo y $\mathrm{V}$ (a) representa el valor de la eficacia global de la acción a (utilizado aquí como el índice global de seguridad en pistas aeroportuarias).

Una vez obtenidos los resultados de eficacia a partir de las evaluaciones locales y globales, se realiza un análisis de los resultados para comprobar si hay valores de eficacia insatisfactoria en uno o varios criterios y analizar las tendencias que pueden comprometer el nivel de seguridad. Este análisis tiene como objetivo identificar los criterios que presentan una eficacia comprometida, lo que requiere acciones de prevención y mitigación de la administración aeroportuaria para devolverlo al nivel de los objetivos de la organización. 
Por último, se recomiendan una serie de acciones a realizar por la administración aeroportuaria con el fin de planificar y devolver los criterios que presentan un nivel de eficacia crítico al nivel de los objetivos de la organización.

\section{RESULTADOS}

Se eligió el Aeropuerto Internacional de São Paulo / Guarulhos como objeto de estudio para ejemplificar la aplicación del enfoque propuesto en este trabajo. Se eligió este aeropuerto por ser el de mayor circulación de aeronaves y pasajeros de América Latina, con una circulación de 284.184 aeronaves y casi 36 millones de pasajeros en 2013.

\section{Construcción y estandarización de la matriz de decisión}

Los diferentes tipos de ESO utilizados como criterios se basaron en el trabajo de Lobianco y Correia (2013) y en la disponibilidad de datos sobre el número de acontecimientos de cada caso, de la siguiente manera:

1) Lesiones corporales $\left(\mathbf{c}_{1}\right)$ : se refiere a una lesión causada por un accidente de trabajo en la plataforma, un accidente con pasajero o un atropellamiento.

2) Ingreso de un animal no volador $\left(c_{2}\right)$ : se refiere a la entrada de un animal no volador en el área de circulación. Por ejemplo, capibaras, perros, caimanes, serpientes.

3) Colisiones entre aeronaves y aves $\left(c_{3}\right)$ : consiste en una colisión de una aeronave con una o más aves, ocurrida en la rampa, en el momento en el que una aeronave está realizando los procedimientos de despegue, aterrizaje o rodaje.

4) Incursión en pista (c4): se produce cuando se da una presencia incorrecta de una aeronave, vehículo o persona en una superficie designada para el aterrizaje o el despegue de una aeronave.

5) Colisión de aeronave en la plataforma $\left(c_{5}\right)$ : se refiere a una colisión en la que se ven implicadas una o más aeronaves sin intención de vuelo. En este indicador se cuentan las colisiones entre una aeronave y un vehículo o entre una aeronave y un equipo cualquiera.

6) Colisión entre propiedades ( $\left.\mathrm{c}_{6}\right)$ : se refiere a una colisión en la que se ven implicados vehículos, equipos u otras propiedades. Este indicador cuenta las colisiones entre: (i) un vehículo o equipo móvil con un edificio; (ii) un vehículo o equipo móvil con cualquier objeto; (iii) dos vehículos o equipos en movimiento; (iv) un vehículo o equipo en movimiento con un vehículo o equipo estacionado; y (v) un vehículo o equipo en movimiento con un vehículo o equipo parado.

7) Desprendimiento de remolque (c7): consiste en un desprendimiento del equipo remolcable (dolly) de su vehículo tractor.

8) Estacionamiento fuera de la zona de seguridad (c8): este indicador cuenta el número de ocasiones en las que una máquina o vehículo se deja fuera de la zona de seguridad y el número de aeronaves estacionadas fuera de su posición de estacionamiento.

9) Objeto extraño en la zona de circulación (c9): este indicador cuenta el número de objetos extraños que se encuentran en el área de circulación. Por ejemplo, la presencia de metales, plásticos, tejidos, poliestireno expandido, hielo, pavimento, papeles, herramientas, madera, globos, cometas.

10) Fugas $\left(c_{10}\right)$ : es la presencia de un derrame de líquido de una aeronave, vehículo u otro equipo. Por ejemplo, derrames de aceites hidráulicos, aceites lubricantes, diesel, combustibles.

Como la eficacia de los criterios se establece en función del número de acontecimientos de los ESO contados en ellos, se tabularon las cifras de acontecimientos anuales de cada evento para la elaboración de la matriz de decisión presentada en la Tabla 1.

En el aeropuerto analizado, se pusieron en circulación respectivamente 154.948, 187.960, 194.184, 209.636 y 250.493 aeronaves en los años 1 a 5. Para reducir las distorsiones en los datos debido a los cambios en la circulación aeroportuaria, se utilizó la Ecuación 1 para calcular el número de ESO producidos al año por cada 100.000 aeronaves en circulación. Los resultados se presentan en la Tabla 2. 
Tabla 1: Número de acontecimientos de cada ESO al año (Lobianco y Correia, 2013)

\begin{tabular}{c|cccccccccc}
\hline Período & $\mathrm{c}_{1}$ & $\mathrm{c}_{2}$ & $\mathrm{c}_{3}$ & $\mathrm{c}_{4}$ & $\mathrm{c}_{5}$ & $\mathrm{C}_{6}$ & $\mathrm{c}_{7}$ & $\mathrm{c}_{8}$ & $\mathrm{c}_{9}$ & $\mathrm{c}_{10}$ \\
\hline Año 1 & 23 & 29 & 27 & 7 & 6 & 21 & 5 & 52 & 16 & 455 \\
Año 2 & 25 & 18 & 11 & 12 & 8 & 21 & 6 & 49 & 15 & 92 \\
Año 3 & 46 & 14 & 28 & 9 & 10 & 25 & 8 & 48 & 16 & 293 \\
Año 4 & 49 & 25 & 44 & 7 & 12 & 40 & 3 & 46 & 23 & 254 \\
Año 5 & 43 & 17 & 27 & 8 & 18 & 71 & 2 & 48 & 26 & 216 \\
\hline
\end{tabular}

Tabla 2: Número de ESO por cada cien mil aeronaves en circulación.

\begin{tabular}{c|cccccccccc}
\hline Período & $\mathrm{c}_{1}$ & $\mathrm{c}_{2}$ & $\mathrm{c}_{3}$ & $\mathrm{c}_{4}$ & $\mathrm{c}_{5}$ & $\mathrm{c}_{6}$ & $\mathrm{c}_{7}$ & $\mathrm{c}_{8}$ & $\mathrm{c}_{9}$ & $\mathrm{c}_{10}$ \\
\hline Año 1 & 14,8 & 18,7 & 17,4 & 4,5 & 3,9 & 13,6 & 3,2 & 33,6 & 10,3 & 294 \\
Año 2 & 13,3 & 9,6 & 5,9 & 6,4 & 4,3 & 11,2 & 3,2 & 26,1 & 8,0 & 49 \\
Año 3 & 23,7 & 7,2 & 14,4 & 4,6 & 5,1 & 12,9 & 4,1 & 24,7 & 8,2 & 151 \\
Año 4 & 23,4 & 11,9 & 21,0 & 3,3 & 5,7 & 19,1 & 1,4 & 21,9 & 11,0 & 121 \\
Año 5 & 17,2 & 6,8 & 10,8 & 3,2 & 7,2 & 28,3 & 0,8 & 19,2 & 10,4 & 86 \\
\hline Neutro & 23,5 & 15,3 & 19,2 & 5,5 & 6,5 & 23,7 & 3,7 & 29,8 & 10,7 & 222,3 \\
Bueno & 14,1 & 7,0 & 8,3 & 3,3 & 4,1 & 12,0 & 1,1 & 20,6 & 8,1 & 67,6 \\
\hline
\end{tabular}

En la siguiente etapa, se definieron los niveles "neutro" y "bueno". Debido a la ausencia de objetivos de seguridad operacionales preestablecidos por la administración aeroportuaria, se utilizaron los cuartiles Q $1 / 4$ y Q3/4 del historial de operación de cada criterio para el cálculo de estos niveles, según lo propuesto por Gonçalves et al. (2014). Los cuartiles $Q_{1 / 4}$ y $Q_{3 / 4}$ se definen como los valores por debajo de los cuales se encuentran, respectivamente, un cuarto y tres cuartos de los datos analizados. Una vez calculados estos niveles (tal y como se muestra en la Tabla 2), se normalizó la matriz de decisión utilizando la Ecuación 2.

\section{Determinación de las tasas de sustitución}

Para determinar las tasas de sustitución, se llevó a cabo una entrevista con un experto en seguridad aeroportuaria, al que se utilizó como responsable de la toma de decisiones en nuestro modelo. Esta entrevista pretendía elaborar una descripción de escenarios pesimistas correspondientes a los ESO en los que se basaba la definición de cada criterio. Los escenarios son los siguientes:

1) Lesión corporal $\left(c_{1}\right)$ : el peor escenario se describió como una lesión seguida de fallecimiento causada por la aspiración de un individuo por toda la unidad motopropulsora de una aeronave, durante la realización de operaciones en rampa.

2) Ingreso de animal no volador ( $\left.\mathrm{c}_{2}\right)$ : el peor escenario se describió como la incursión de un buey adulto (peso medio de $450 \mathrm{~kg}$ ) en la pista de aterrizaje / despegue, durante una operación de despegue de un Boeing 747-800 a su máxima capacidad de pasajeros y combustible.

3) Colisión entre aeronave y ave $\left(c_{3}\right)$ : el peor escenario fue descrito como una colisión entre un Boeing 747 800 y varios buitres de cabeza negra adultos (las aves más grandes de la fauna local), producido durante la etapa del proceso de despegue en la que el avión alcanza la velocidad máxima.

4) Incursión en pista $\left(\mathrm{c}_{4}\right)$ : el peor escenario se describió como una incursión en la pista de un Boeing 747 800 a 30 km / h (máxima velocidad de rodaje permitida) durante una operación de despegue de otro Boeing 747-800, considerando que ambos se encontraban a su máxima capacidad de pasajeros (467 personas) y combustible (238,610 litros).

5) Colisión de aeronave en la plataforma (c5): el peor escenario se describió como una colisión frontal entre un Boeing 747-800 y un camión cisterna. En este escenario, ambos vehículos se estarían moviendo a $30 \mathrm{~km}$ / h, que es la velocidad máxima permitida en la vía, y a su capacidad máxima de pasajeros (467 personas) y combustible (238,610 litros). 
6) Colisión entre propiedades $\left(\mathrm{c}_{6}\right)$ : el peor escenario para este evento se describió como una colisión frontal entre un autobús (a su capacidad máxima de pasajeros) y un camión cisterna, en el que ambos vehículos circulaban a la velocidad máxima permitida en la vía, de $30 \mathrm{~km} / \mathrm{h}$.

7) Desprendimiento de remolque ( $\left.c_{7}\right)$ : para este evento, el peor de los escenarios se describió como el desprendimiento de un remolque de transporte de queroseno a $30 \mathrm{~km} / \mathrm{h}$ en una pista con una pendiente del $1 \%$.

8) Estacionamiento fuera de la zona de seguridad (c8): el peor de los escenarios se describió como el estacionamiento de una aeronave dentro de una vía de circulación sin señalización adecuada que indique su presencia y durante el rodaje de otras aeronaves por la vía.

9) Objeto extraño en la zona de circulación (c9): el peor de los escenarios se describió como la presencia de una llave dinamométrica abandonada en la zona de circulación, lo que supondría una herramienta de metal de hasta 16 kilogramos.

10) Fugas ( $\left.c_{10}\right)$ : el peor escenario se describió como una fuga de 238,610 litros de combustible aeronáutico, lo que se corresponde con la capacidad máxima de almacenamiento de combustible de un Boeing 747-800.

Una vez caracterizados los ESO correspondientes a cada criterio en el peor escenario posible, se utilizaron los mismos como base para la obtención de pesos, lo cual se realizó por el método Swing Weights. En la primera etapa del método Swing Weights, se le pidió al responsable de la toma de decisiones que considerase el suceso de cada criterio en su peor escenario, haciéndole la siguiente pregunta: "Suponiendo que todos los criterios presenten una eficacia en el nivel "neutro", y teniendo la posibilidad de mejorar un único criterio hasta el nivel "bueno", ¿qué criterio elegiría para alcanzar una mayor mejora de la seguridad aeroportuaria?" Como respuesta, el responsable de la toma de decisiones ofreció el criterio c4. En el siguiente paso del método Swing Weights, se preguntó al responsable de la toma de decisiones: "Sin tener en cuenta el criterio ofrecido en la pregunta anterior, ¿qué otro criterio elegiría para mejorar, pasando del nivel "neutro" al nivel "bueno", a fin de alcanzar una mayor mejora de la seguridad?" Como respuesta, el responsable de la toma de decisiones ofreció el criterio $c_{5}$. Este procedimiento se repitió hasta que el responsable de la toma de decisiones hubo ordenado todos los criterios, lo que resultó en el orden de $\mathrm{C}_{4}$, $\mathrm{C}_{5}$, $\mathrm{C}_{6}, \mathrm{C}_{3}, \mathrm{C}_{1}, \mathrm{C}_{2}, \mathrm{C}_{8}, \mathrm{C}_{10}, \mathrm{C}_{7}$ y $\mathrm{C}_{9}$, tal y como se ilustra en la Fig. 2.

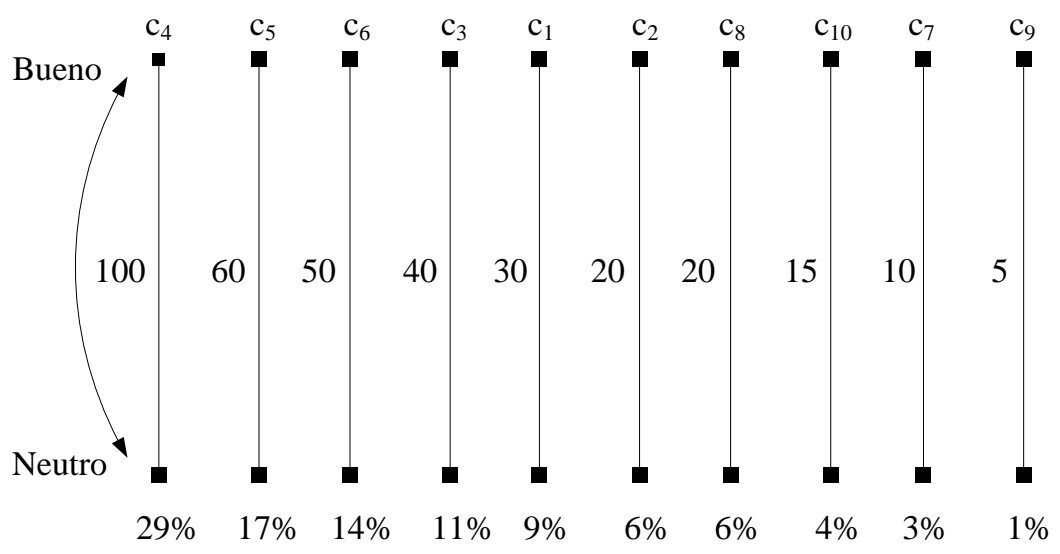

Fig. 2: Determinación de las tasas de sustitución con el método Swing Weights.

Una vez ordenados los criterios, se pidió al responsable de la toma de decisiones que los puntuase en relación al riesgo que cada uno supone para la seguridad. En base a una puntuación de 100 para el criterio de mayor importancia (c4), se preguntó al responsable de la toma de decisiones sobre el resultado de los demás criterios con puntuaciones de 60, 50, 40, 30, 20, 20, 15, 10 y 5. Estos valores se estandarizaron, lo cual dio como resultado los pesos ilustrados en porcentajes en la Fig. 2.

\section{Evaluación de la eficacia global, local y análisis de sensibilidad}

Para llevar a cabo las evaluaciones de eficacia, se utilizaron la Ecuación 2 y los datos de la Tabla 2 para calcular los valores de eficacia local de cada criterio, tal y como se muestra en la Tabla 3. Además, se utilizaron la Ecuación 3 y las tasas de sustitución presentadas en la Fig. 2 para calcular los valores de eficacia global. Pueden consultarse los niveles de eficacia local y global en la Tabla 3. 
Tabla 3: Matriz de decisión estandarizada y datos de tendencia.

\begin{tabular}{|c|c|c|c|c|c|c|c|c|c|c|c|}
\hline Período & $\mathrm{v}\left(\mathrm{c}_{1}\right)$ & $\mathrm{v}\left(\mathrm{c}_{2}\right)$ & $\mathrm{v}\left(\mathrm{c}_{3}\right)$ & $\mathrm{v}\left(\mathrm{c}_{4}\right)$ & $\mathrm{v}\left(\mathrm{c}_{5}\right)$ & $\mathrm{v}\left(\mathrm{c}_{6}\right)$ & $\mathrm{v}\left(\mathrm{c}_{7}\right)$ & $\mathrm{v}\left(\mathrm{c}_{8}\right)$ & $\mathrm{v}\left(\mathrm{c}_{9}\right)$ & $\mathrm{v}\left(\mathrm{c}_{10}\right)$ & $\mathrm{V}(\mathrm{a})$ \\
\hline Año 1 & 92,6 & $-41,0$ & 16,5 & 45,5 & 108,3 & 86,3 & 19,2 & $-41,3$ & 15,4 & $-46,3$ & 41,6 \\
\hline Año 2 & 108,5 & 68,7 & 122,0 & $-40,9$ & 91,7 & 106,8 & 19,2 & 40,2 & 103,8 & 112,0 & 77,4 \\
\hline Año 3 & $-2,1$ & 97,6 & 44,0 & 40,9 & 58,3 & 92,3 & $-15,4$ & 55,4 & 96,2 & 46,1 & 42,1 \\
\hline Año 4 & 1,1 & 41,0 & $-16,5$ & 100,0 & 33,3 & 39,3 & 88,5 & 85,9 & $-11,5$ & 65,5 & 30,4 \\
\hline Año 5 & 67,0 & 102,4 & 77,1 & 104,5 & $-29,2$ & $-39,3$ & 111,5 & 115,2 & 11,5 & 88,1 & 66,7 \\
\hline$\alpha$ & $-15,9$ & 25,9 & $-1,7$ & 25,9 & $-33,3$ & $-31,9$ & 25,4 & 35,9 & $-12,3$ & 22,2 & 0,3 \\
\hline $\mathrm{R}^{2}$ & 0,2 & 0,5 & 0,0 & 0,5 & 0,9 & 0,7 & 0,6 & 0,9 & 0,1 & 0,3 & 0,0 \\
\hline
\end{tabular}

La evaluación de la eficacia local se inició mediante el análisis de la eficacia de los criterios en el año 5 , ya que, al ser más recientes, se consideraron más representativos de la realidad aeroportuaria. El año 5 presentó cuatro criterios con niveles de eficacia excelentes $\left(c_{2}, c_{4}, c_{7}\right.$ y $\left.c_{8}\right)$, cuatro con un nivel de eficacia competitivo $\left(c_{1}, c_{3}, c_{9}\right.$ y $\left.c_{10}\right)$ y dos con un nivel de eficacia comprometedor $\left(c_{5}\right.$ y $\left.c_{6}\right)$. Cabe destacar que la eficacia del criterio $c_{9}$ presentó una puntuación competitiva, pero próxima al nivel de impacto comprometedor, por lo que resultaría coherente planificar acciones para mejorar su eficacia.Además, se calcularon el coeficiente angular de la pendiente de regresión $(\alpha)$ y el coeficiente de determinación $\left(R^{2}\right)$ para analizar la existencia o ausencia de tendencias descendentes en los valores de la eficacia de cada criterio. El coeficiente a representa el declive de la pendiente de regresión lineal, siendo positivo cuando la pendiente muestra una función ascendente y negativo cuando es descendente. En cuanto al coeficiente $\mathrm{R}^{2}$, se trata de una medida de ajuste del modelo estadístico lineal que varía de cero a uno e indica en porcentajes hasta qué punto un modelo de regresión explica los valores de cada criterio. Pueden consultarse los valores de $\alpha$ y $\mathrm{R}^{2}$ en la Tabla 3.

Para identificar las tendencias descendentes, se analizaron los criterios que mostraban un alto grado de ajuste al modelo de regresión y los valores negativos de $\alpha$, de tal manera que se identificaron tendencias descendentes en los criterios $\mathrm{c}_{5}$ y $\mathrm{c}_{6}$. Por otra parte, pudo constatarse que estos criterios también tuvieron una eficacia crítica en el año 5 . En este sentido, se verificó la necesidad de supervisarlos y planificar acciones para reducir y mitigar los riesgos derivados de ellos.

Al analizar el historial de la eficacia global, se observó que la eficacia de la seguridad operacional del aeropuerto se mantuvo en la zona comprendida entre los niveles "neutro" y "bueno" en todos los años evaluados, alcanzándose el mejor resultado global en el año 2, seguido por los años 5, 3, 1 y 4 respectivamente. Este análisis indica que la eficacia global no ha mantenido una tendencia de mejora continua, lo cual queda evidenciado por la disminución de la eficacia producida en los años 3 y 4 . Además, el año 5 presentó una disminución de un 13,8 \% con respecto al valor alcanzado en el año 2, el cual mostró el mayor nivel de eficacia durante el período estudiado. Una vez evaluados los niveles de eficacia local y global, se realizó un análisis de sensibilidad variando las tasas de sustitución en $-10 \%$ y $+10 \%$ en cada criterio individual. Incluso con estas alteraciones, no hubo cambios significativos en el valor de la eficacia global de cada año del historial evaluado, y ninguno de los valores de eficacia globales alcanzó una puntuación comprometedora.

\section{DISCUSIÓN}

En esta sección se presentan las recomendaciones para la gestión aeroportuaria con el fin de planificar acciones para que los criterios con un nivel de eficacia crítico puedan devolverse al nivel de los objetivos organizacionales. En este contexto, se analizaron los criterios $\mathrm{c}_{5}, \mathrm{c}_{6}$ y $\mathrm{c}_{9}$.

En cuanto al criterio $c_{5}$ (colisión de aeronave en la plataforma), al analizar los datos de seguridad aeroportuaria, se concluyó que la mayoría de los sucesos se deben a la falta de atención de los operadores, habiendo también un porcentaje de sucesos producidos por fallos de los equipos, fallos de procedimiento e incumplimiento de las normas. En este sentido, se recomendó la adopción de las siguientes medidas: realización de una inspección / revisión anual (técnica y documental) de los equipos y vehículos que ingresan en las plataformas y pistas, desarrollo de políticas de inspecciones de rutina por los inspectores de plataformas, mejora de la señalización (por ejemplo: conos reflectantes) para marcar los límites extremos de la zona de estacionamiento de cada aeronave, realización de sesiones de capacitación que hagan hincapié en la importancia de prestar atención al movimiento de los vehículos y equipos en las proximidades de las aeronaves, realización de sesiones de capacitación sobre las normas operacionales de la plataforma y realización de operaciones de mantenimiento preventivo de vehículos y equipos. 
En cuanto al criterio $c_{6}$ (colisión entre propiedades), se observó que la mayoría de las colisiones registradas fueron causadas por la falta de atención de sus conductores, por el incumplimiento de las normas, por fallos de equipos y fallos de procedimientos. Como resultado, además de las medidas recomendadas para el criterio $\mathrm{C}_{1}$, se recomiendan las siguientes acciones: realización de cursos de actualización y perfeccionamiento para conductores implicados en incidentes, advertencias concretas colocadas en lugares de gran circulación y control del movimiento de vehículos por medio de etiquetas electrónicas similares a las que se utilizan en los peajes.

Con respecto al criterio $c_{9}$ (objeto extraño en el área de movimiento), que se refiere principalmente a los incidentes y accidentes ocurridos como consecuencia de la ingestión de un objeto por los motores o de la colisión del mismo contra una aeronave, se propusieron las siguientes acciones para mejorar su eficacia: aumentar la frecuencia con la que se llevan a cabo las inspecciones en las pistas y pistas de rodaje para la retirada de objetos extraños, impulsar campañas de sensibilización sobre las consecuencias de la presencia de objetos extraños para toda la comunidad aeroportuaria y trabajar con los subcontratistas para que reevalúen sus procedimientos con el fin de aumentar la seguridad operacional del aeropuerto.

\section{CONCLUSIONES}

La aplicación del enfoque propuesto generó resultados que la administración aeroportuaria consideró coherentes y precisos, lo que demuestra que la sólida base teórica de la MAVT en la teoría del valor ofrece el potencial para lograr mejores resultados que las metodologías arbitradas o desarrolladas de forma empírica.

El procedimiento propuesto para la ponderación de los criterios posibilitó que el proceso de modelado contase con mayor consistencia, lo que a su vez contribuyó a que se pudieran eludir las limitaciones de los trabajos realizados anteriormente, que utilizaron datos ordinales en este cálculo. El uso del método Swing Weights fue considerado sencillo y consistente, ya que permite calcular los pesos de forma transparente para la gestión aeroportuaria.

Gracias al uso de los cuartiles $Q_{1 / 4}$ y $Q_{3 / 4}$ se pudo verificar hasta qué punto la eficacia obtenida en la evaluación de la seguridad operacional alcanzó los objetivos de la administración aeroportuaria, con el fin de facilitar el control de los resultados y la identificación de urgencias. Por otra parte, los indicadores de eficacia de la seguridad operacional se podrían utilizar para identificar y evaluar situaciones de alto riesgo, lo que ayudaría a priorizar las acciones encaminadas a reducir y mitigar los riesgos con el fin de ahorrar tiempo y recursos.

\section{AGRADECIMIENTOS}

Los autores agradecen al Consejo Nacional de Desarrollo Científico y Tecnológico- CNPq el apoyo prestado para la realización de esta investigación.

\section{REFERENCIAS}

Ahn, B. S., Compatible weighting method with rank order centroid: Maximum entropy ordered weighted averaging approach, European Journal of Operational Research, 212(3), 552-559, (2011)

Allford, L., Process safety indicators: Response to Andrew Hopkins, Safety Science, 47(4), 466 (2009)

ANAC - Agencia Nacional de Aviación Civil de Brasil, Programa de Seguridad Operacional Específico de la Agencia Nacional de Aviación Civil (PSOE-ANAC), 32, Brasilia, Brasil (2009)

Barron, F. H. y B. E. Barrett, The efficacy of SMARTER - Simple multi-attribute rating technique extended to ranking, Acta Psychologica, 93, 23-36 (1996)

Basso, B., Carpegna, C., Dibitonto, C., Gaido, G., Robotto, A. y C. Zonato, Reviewing the safety management system by incident investigation and performance indicators, Journal of Loss Prevention in the Process Industries, 17(3), 225-231 (2004)

Belton, V., "Multi-criteria problem structuring and analysis in a value theory framework", en Multicriteria decision making: Advances in MCDM models, algorithms, theory, and its applications, T. Gal, T. J. Stewart y T. Hanne, Kluwer Academic, Boston, USA (1999) 
BOEING, "Statistical Summary of Commercial Jet Airplane Accidents - Worldwide Operations: 1959 - 2010" (en línea), 11 de septiembre de 2014, http://www.boeing.com/news/techissues/pdf/statsum.pdf, BOEING, USA (2011)

Campos, J., Taboada, C. y Chalmeta, R. Metodología para la Evaluación del Rendimiento de la Cadena Logística, Información Tecnológica, 15(4), 77-84 (2004)

Edwards, W. y F. H. Barron, "SMARTS and SMARTER: Improved simple methods for multiattribute utility measurement”, Organizational Behavior and Human Decision Processes, 60(3), 306-325 (1994)

FAA - Administración Federal de Aviación de Estados Unidos, "AC 120-90 - Line Operations Safety Audits Document Information" (en línea), 11 de septiembre 2014, http://www.faa.gov/regulations_policies/advisory_circulars/ index.cfm/go/document.information/documentID/22478, AFS-230, USA (2006)

Gonçalves, T. J. M., Correia, A. R. y J. F. B. Lobianco, Multicriteria approach to evaluate airport safety levels, presentado en la 18ª Conferencia Mundial de la Air Transport Research Society, Burdeos, Francia (2014)

Goodwin, P. y G. Wright, Decision analysis for management judgment, John Wiley \& Sons, Londres, Reino Unido (2009)

Hale, A., Special issue on process safety indicators, Safety Science, 47(4), 459 (2009)

Hopkins, A., Thinking about process safety indicators Safety Science, 47(4), 460-465 (2009a)

Hopkins, A., Reply to comments, Safety Science, 47(4), 508-510 (2009b)

ICAO - International Civil Aviation Organization, Doc 9859 - Safety Management Manual (SMM). 284, Montréal, Canadá (2009)

Janaćković, G., Savić, S., y M. Stanković, Multi-criteria decision analysis in occupational safety management systems. safety engineering, 1(1), 17-22 (2011)

Lobianco, J. F. B. y A. R. Correia, Methodology to obtain airport safety indicators using safety management systems, Journal of the Brasilian Air Transportation Research Society, 9(1), 47-58 (2013)

Matilla, M. M. y Chalmeta, R. Metodología para la Implantación de un Sistema de Medición del Rendimiento Empresarial, Información Tecnológica, 18(1), 119-126, (2007)

Montibeller, G., Gummer, H. y D. Tumidei, Combining scenario planning and multi-criteria decision analysis in practice, Journal of Multi-Criteria Decision Analysis, 14, 5-20, (2006)

Øien, K., Utne, I. B. y I. A. Herrera, Building safety indicators: Part 1 - Theoretical foundation, Safety Science, 49(2), 148-161 (2011)

Reason, J., Managing the risks of organizational accidents, $1^{\text {a }}$ ed., 252, Ashgate, Burlington, EE.UU. (1997)

Roelen, A. L. C. y M. B. Klompstra, The challenges in defining aviation safety performance indicators, Presentado en la 11 a Conferencia de International Probabilistic Safety Assessment and Management y la Conferencia annual de European Safety and Reliability, Helsinki, Finlandia (2012)

Wreathall, J., Leading? Lagging? Whatever!, Safety Science, 47(4), 493-494 (2009) 